\title{
Investigation of double-gap cavity generating-amplifying klystrons
}

\author{
Tatyana Remizova, Valeriy Fedyaev, Basil Yurkin \\ The Ryazan State Radio University, RGRTU, Faculty of Electronic, Ryazan, Russia \\ Email address: \\ tatyanaremizova2009@yandex.ru(T. Remizova), fedyaev.v.k@rsreu.ru(V.Fedyaev), yurkin_vi@inbox.ru(B. Yurkin)
}

\section{To cite this article:}

Tatyana Remizova, Valeriy Fedyaev, Basil Yurkin. Investigation of Double-Gap Cavity Generating-Amplifying Klystrons. International Journal of Energy and Power Engineering. Vol. 2, No. 3, 2013, pp. 104-108. doi: 10.11648/j.ijepe.20130203.13

\begin{abstract}
Based upon the discrete model of large particle flux investigated is the possibility of operation of a double-gap cavity resonator in the mode where the oscillation generating function is aligned with the function of velocity-modulating electrons in the phase ensuring further sufficient electron bunching. It is established that the required distribution of velocities is gained on the edge of the generation area with longest distance between gaps where both phase and amplitude conditions of self-excitement are met. Shown is the possibility of obtaining the highest efficiency at the expense of using a double-gap cavity resonator with wide gaps. Performed is the investigation of generating-amplifying klystrons of different configurations. Found are conditions of obtaining a maximum value of amplitude of the convection current first harmonic and a maximum value of the efficiency factor.
\end{abstract}

Keywords: Double-Gap Cavity, Generating-Amplifying Klystron, Efficiency Factor

\section{Introduction}

The innovation mechanism of the modern power industry is integrally connected with establishment and employment of high-efficiency energy sources. Microwave energy primarily belongs to such sources as in a number of uses it has undeniable advantages over others: it brings no contamination during heating, virtually inertialess in control.

Recently Russia and foreign nations initiated activities on creating simple-configuration microwave power generators with a high efficiency factor for industrial, energy, medical and technology purposes. There are many published works dedicated to microwave generators based on one resonator with one wide gap [1, 2] and with two gaps[3 - 5].

The known disadvantage of generators the resonant system of which is connected through the energy output with a load is impact of transformed complex load resistance to the frequency and power of oscillations generated, i.e. a frequency pulling effect.

In the 1950s Harrison[6] and Warneke[7] in their works proposed an idea of isolating a generator section from the output stage. In this particular case, the load changes bringing about the output resonator detuning cannot directly impact the oscillations frequency which is determined by the generator itself. In the work[8] the idea was used for decoupling from the generator section load on the double-cavity closed-cycle klystron. Such a configuration got a name of klystron with a buffer amplifier.

In that case, the given idea was used for decoupling of the generator section from a load on one double-gap cavity resonator. The operation mode of the double-gap cavity resonator in the generator section will differ from the operation mode of such a resonator in amplifying klystrons and from the operation mode as a generator. The generator section should combine the functions of generation and velocity-modulation of electrons in the required phase. It is necessary to select such conditions under which in the generation mode at the output of the double-gap cavity resonator electron bunches are formed with distribution of velocities ensuring further densification of the bunch, its grouping and increase of the convection current first harmonic. In that case, in placing gaps of the output resonator in the area of maximum growing convection current first harmonic the efficiency factor is increased as compared with the efficiency of a generator without a buffer stage. At that the generator section will be completely decoupled from the load the change of which will not be able to impact the oscillations frequency.

In work [9] it is shown that after a generator section the electron grouping mode is possible if the length of the drift tube between gaps is increased as compared with its length 
for the generation area centre. The calculations were executed in the kinematic approximation[10].

In the given work with use of the program[11] based on a five-layer model of the electron flux of deformed elements investigated in a self-coordinated mode is a possibility of increasing the efficiency factor in the following configurations of generating-amplifying klystrons:

- In a three-cavity double-gap generating-amplifying klystron of the four-centimetre band where the generator section is stabilized by a high-Q cavity resonator and completely decoupled from the load with an intermediate stage[12]. The efficiency increase was achieved by altering the resonators interaction area only without changing other structural dimensions.

- In a two-cavity double-gap generating-amplifying klystron of the decimetre band[13] at the expense of changing the accelerating voltage and current of the beam.

- In a two-cavity generating-amplifying klystron of the three-centimetre band with a generator section on a two-gap cavity resonator with wide gaps and an output one-gap resonator.

In a self-coordinated mode the voltages at the resonator gaps are determined by an iteration method[14, 15] via the resonators' induced current and effective resistance.

Thus, the present article investigates the specified changed operation mode of the double-gap resonator while aligning the oscillations generation and velocity modulation of electrons in the phase ensuring further grouping of electron bunches. Shown are the results of investigating the possibility of increasing the convection current first harmonic in generating-amplifying klystrons at the expense of bunching electrons after the generator section. According to preliminary calculations the efficiency factor of such generating-amplifying klystrons constitutes about 50-70\% for different designs.

\section{Simulation Results}

The results of calculating such generators with a buffer stage are shown in table (1) and table (2).

The interaction of electron flux with fields of microwave gaps in the dynamic mode was investigated by a method of a self-coordinated field taking into consideration the impact of resonator parameters, i.e. characteristic resistances and quality factors to the process of buildup and settling of microwave energy oscillations. According to the program of two-dimensional analysis with consideration of spatial charge optimality calculations were conducted to the maximum efficiency factor in output resonators of generating-amplifying klystrons. The design of the resonator instrument unit is shown in figure (1).

As a result of parametric optimization we simulated a mode of a continuously gathering bunch with a continuous growth of convection current along the investigated instruments (figure 2). However, in a definite area of the second gap of the tristron's[13] output resonator (figure 1,b) and klystron's input resonator (figure 1,c) the alternating voltage has an unbunching effect on the electron flux (figure 2,b) which brings about an occurrence of a small fall (figure 2,f) and characteristic break of the current curve (figure 2,e).

Table 1. Design data of instruments

\begin{tabular}{|c|c|c|c|c|c|c|c|c|c|c|c|c|c|c|c|c|c|c|c|c|c|c|c|}
\hline \multirow{2}{*}{\multicolumn{2}{|c|}{$\begin{array}{c}\text { № } \\
\text { Instrument } \\
\text { Resonator } \\
\text { Gap } \\
\end{array}$}} & \multicolumn{3}{|c|}{$\begin{array}{l}\text { Gap length d, } \\
\text { rad }\end{array}$} & \multicolumn{3}{|c|}{$\begin{array}{c}\text { Drift tunnel l, } \\
\text { rad }\end{array}$} & \multicolumn{3}{|c|}{$\begin{array}{c}\text { Resonator length } \\
\text { h, rad }\end{array}$} & \multicolumn{3}{|c|}{$\begin{array}{l}\text { Centre distance } \\
\text { of resonators } l_{c} \text {, } \\
\operatorname{rad}\end{array}$} & \multicolumn{3}{|c|}{$\begin{array}{c}\text { Number of beam } \\
\text { of current }\end{array}$} & \multirow{2}{*}{$\begin{array}{c}\text { Beam } \\
\text { radius, } \\
\text { rad } \\
1\end{array}$} & \multicolumn{3}{|c|}{$\begin{array}{c}\text { Canal radius, } \\
\text { rad }\end{array}$} & \multicolumn{3}{|c|}{ Block coefficient } \\
\hline & & 1 & 2 & 3 & 1 & 2 & 3 & 1 & 2 & 3 & 1 & 2 & 3 & 1 & 2 & 3 & & 1 & 2 & 3 & 1 & 2 & 3 \\
\hline \multirow{2}{*}{1} & 1 & 1.7 & 1.5 & 4.8 & \multirow{2}{*}{7.2} & \multirow{2}{*}{4.2} & \multirow{2}{*}{3.6} & \multirow{2}{*}{13.2} & \multirow{2}{*}{8.9} & \multirow{2}{*}{15.9} & \multirow{2}{*}{14.2} & \multirow{2}{*}{8.1} & \multirow{2}{*}{11.5} & \multirow[b]{3}{*}{7} & \multirow[b]{3}{*}{4} & \multirow[b]{3}{*}{6} & \multirow[b]{3}{*}{1} & \multirow[b]{3}{*}{4.5} & \multirow[b]{3}{*}{0.7} & \multirow[b]{3}{*}{0.4} & \multirow[b]{3}{*}{0.6} & \multirow[b]{3}{*}{0.6} & \multirow{4}{*}{0.5} \\
\hline & 2 & 1.7 & 1.5 & 4 & & & & & & & & & & & & & & & & & & & \\
\hline 2 & $\begin{array}{l}3 \\
4\end{array}$ & $\begin{array}{l}2 \\
2\end{array}$ & $\begin{array}{l}1.8 \\
1.8\end{array}$ & 0.7 & 1.1 & 1.8 & & 8.4 & 7.3 & 4.2 & 10.2 & & & & & & & & & & & & \\
\hline 3 & $\begin{array}{l}5 \\
6\end{array}$ & $\begin{array}{l}1.4 \\
1.4\end{array}$ & & & 0.8 & & & 8.4 & & & & & & & & & & & & & & & \\
\hline
\end{tabular}

Table 2. Physical properties of instruments

\begin{tabular}{|c|c|c|c|c|c|c|c|c|c|c|c|c|c|c|c|c|c|}
\hline \multirow{2}{*}{$\begin{array}{c}\begin{array}{c}\text { Resonat } \\
\text { or } \\
\text { Instrument }\end{array} \\
\end{array}$} & \multicolumn{3}{|c|}{$\begin{array}{l}\text { Equivalent } \\
\text { resistance }\end{array}$} & \multicolumn{3}{|c|}{$\begin{array}{c}\text { Voltage amplitude, } \\
\qquad \mathrm{kV}\end{array}$} & \multicolumn{3}{|c|}{$\begin{array}{c}\text { Electron } \\
\text { efficiency of } \\
\text { resonators, \% }\end{array}$} & \multirow{2}{*}{$\begin{array}{c}\text { Phase } \\
\text { shift } \\
\varphi_{I^{\wedge} U}, \\
\text { rad } \\
2\end{array}$} & \multirow{2}{*}{$\begin{array}{c}\text { Maximu } \\
\text { m voltage } \\
\text { of } \\
\text { convection } \\
\text { current } \\
\mathbf{I}_{1 \mathrm{KON} \max }, \mathrm{A} \\
\end{array}$} & \multirow{2}{*}{$\begin{array}{l}\text { Wave } \\
\text { length } \\
\lambda, \mathrm{cm}\end{array}$} & \multirow{2}{*}{$\begin{array}{c}\text { Beam } \\
\text { current } \\
\mathbf{I}_{0}, \mathbf{A}\end{array}$} & \multirow{2}{*}{$\begin{array}{c}\text { Accelerating } \\
\text { voltage } \mathbf{U}_{\mathbf{0}}, \\
\mathbf{k V}\end{array}$} & \multirow{2}{*}{$\begin{array}{c}\text { Input } \\
\text { power, } \\
\text { MW }\end{array}$} & \multirow{2}{*}{$\begin{array}{c}\text { Output } \\
\text { power, } \\
\text { MW }\end{array}$} & \multirow{2}{*}{$\begin{array}{l}\text { Efficiency } \\
\text { factor, \% }\end{array}$} \\
\hline & 1 & 2 & 3 & 1 & 2 & 3 & 1 & 2 & 3 & & & & & & & & \\
\hline 1 & 2.2 & 8.9 & 0.4 & 0.1 & 0.14 & 0.46 & 0.5 & 0.2 & 50 & & 0.02 & 4.3 & 0.01 & 0.7 & $5 \cdot 10^{-5}$ & $2.4 \cdot 10^{-5}$ & 48 \\
\hline 2 & 2.8 & 1.4 & & 1.65 & 6.6 & & 1.6 & 55 & & 1.55 & 0.2 & 56.6 & 0.12 & 5.5 & $3 \cdot 10^{-3}$ & $1.4 \cdot 10^{-3}$ & 52 \\
\hline 3 & 13.5 & 0.2 & & 285 & 275 & & 0.8 & 70 & & & 58 & 3 & 37.5 & 250 & 37.5 & 25.6 & 69 \\
\hline
\end{tabular}




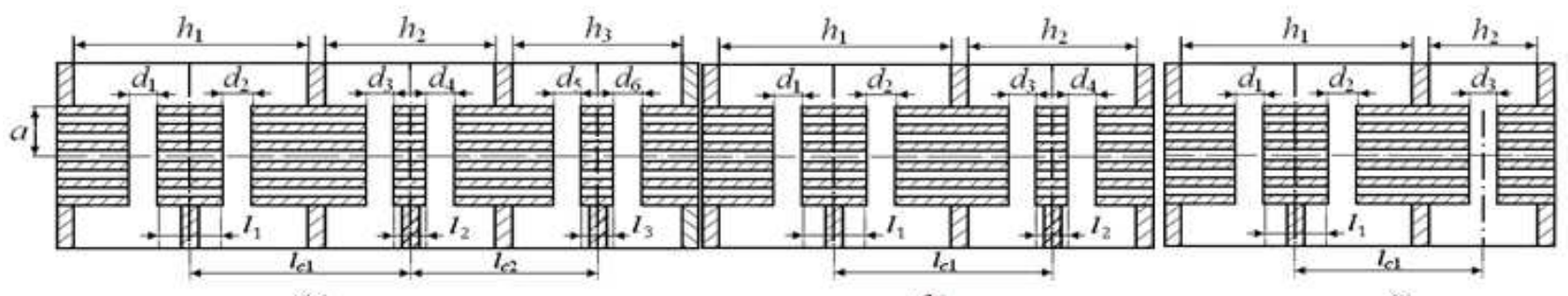

(a)

(b)

(c)

Fig 1 . The design of the resonator in a three-cavity generating-amplifying klystron (a), tristron's (b), two-cavity generating-amplifying klystron (c)
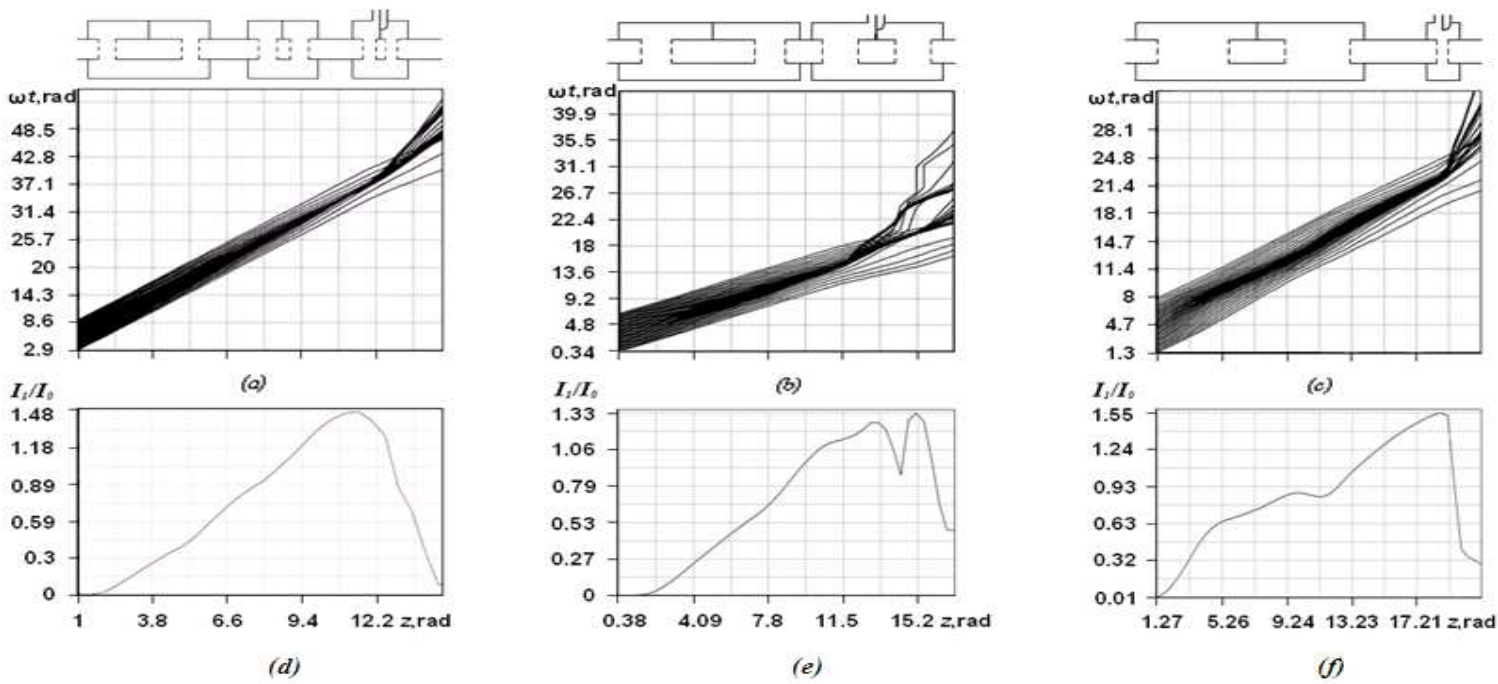

Fig 2. Space-time diagram of electron motion and amplitude of a first harmonic of convection current: (a), (d) - three-cavity generating-amplifying klystron; (b), (e) - tristron's; (c), (f) - two-cavity generating-amplifying klystron

To obtain a maximum efficiency factor on the load power at a numerical simulation of electronic processes in a three-resonator configuration of the generating-amplifying klystron in comparison with the sample manufactured the dimensions of interaction areas of the resonators were altered. In the first resonator the distance between gap middles was increased to the edge of the first generation area. The dimensions of gaps of the first and second resonators were left without changes. The velocity modulation phase was gained with such a value that here in the drift tube between the first and second resonator the complete electron bunching occurred. In the third resonator tuned to the frequency of generated oscillations the length of gaps was reduced. The distance between the gap centre's of the second and third resonators was also under reduction. All resonators work with the $\pi$-type of oscillations.

The configuration of the two-gap two-resonator tristron was left without alterations. In that case to ensure the condition of complete electron bunching the beam accelerating voltage and current were changed. The tristron's resonators work with the 0 -type of oscillations.

The calculations executed showed that the use of the generator section on the resonator with two narrow gaps ensuring the phase condition of the complete grouping of the bunches previously grouped in the resonator drift tube and increase of the convection current first harmonic amplitude does not give high values of the efficiency factor.

The purpose of the present work is creation of a source of microwave healing of a simple configuration with a high efficiency factor. Thus, the microwave oscillation generator with a generator section on a double-gap resonator with wide gaps and a buffer stage on a one-gap resonator with a narrow gap was engineered (figure 1,c). As a result of calculations at selected optimal instrument parameters with consideration of the magnetic field an efficiency factor of about $70 \%$ was gained. As with a formal optimization the physical processes are hidden a portion of results is presented in the article in the form of functional relationships.

For visual analysis of electron grouping processes calculations were executed in the developing two-cavity generator on the graphical program of representing information which uses numerical data obtained according to a two-dimensional program in building different graphical relationships. Figure (3) shows diagrams of relationships of longitudinal normalized velocities of electrons (marked by dots) from the phase of their arrival at different planes along the instrument. The normalization of electron velocities is made with the constant component of electron velocities. By means of the given diagrams we will conduct a detailed analysis of the process of grouping electrons and changing their velocities. 
In the generation mode in the first gap of the input resonator working with the $\pi$-type of oscillations the velocity modulation of the electron flux is executed. Electrons leaving the gap earlier are accelerated by the gap microwave voltage field and those leaving later are decelerated by that voltage (figure $3, a$ ). At the expense of this in the resonator drift tube prebunching of the electron flux and accordingly reduction of the electron velocity scatter occurs (figure 3,b). The forming bunch with execution of the self-excitement phase condition gives off a portion of its energy to the field of the output resonator second gap. In the generation mode the gap velocity modulation phase will be at such a value that electrons of the bunch flying at the front are also decelerated and those at the back are accelerated (figure 3,c). Then in the drift tube of the buffer stage an additional densification of the bunch will occur (figure 3,d).

It can be clearly seen on the space-time diagram (figure2,c) how in the optimized version the idea of aligning the function of generating oscillations and velocity-modulating electrons in the required phase in the double-gap resonator is implemented: with the positive

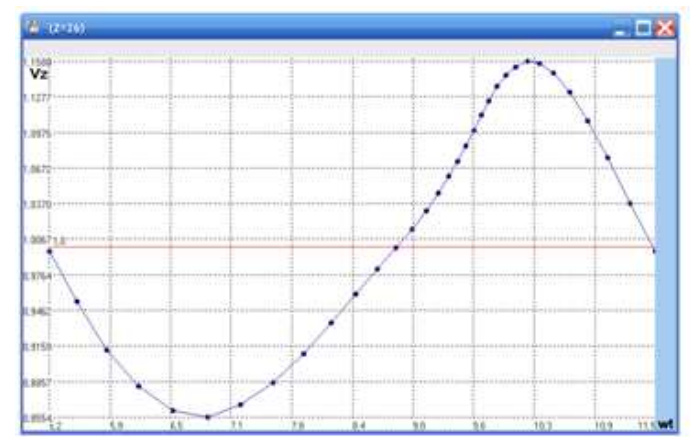

(a)

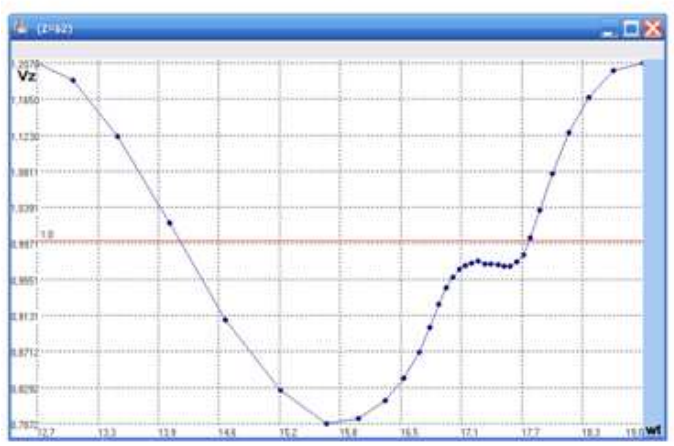

value of the electronic efficiency of the generator section one can see at the whole length of the instrument a gathering bunch with an almost unchangeable bunching centre. The intensive bunching of electrons occurs already in the first long gap: the current amplitude at the gap output constitutes $0.63 \mathrm{I}_{0}$. In the drift tube of the generator section the current grows to a value of $0.8 \mathrm{I}_{0}$. In the second gap where energy is taken from the electron flux the current harmonic value slightly decreases but in the second drift tube the bunching continues and the current harmonic grows to a value of $1.55 \mathrm{I}_{0}$ (figure $2, \mathrm{f}$ ). In the gap of the amplitude resonator with a voltage amplitude of $1.1 \mathrm{U}_{0}$ energy is taken from the bunch with an electronic efficiency of $70 \%$. It is unbunched and the current harmonic value falls virtually to zero.

In analyzing electronic processes it is often necessary to know the exact value of coordinates and velocities of separate electrons. By means of diagrams of functional relationships of longitudinal (figure 4,a) and radial (figure $4, \mathrm{~b})$ normalized velocities of electrons from the longitudinal coordinate one can trace the changes of velocities of separate electrons along the interaction space.

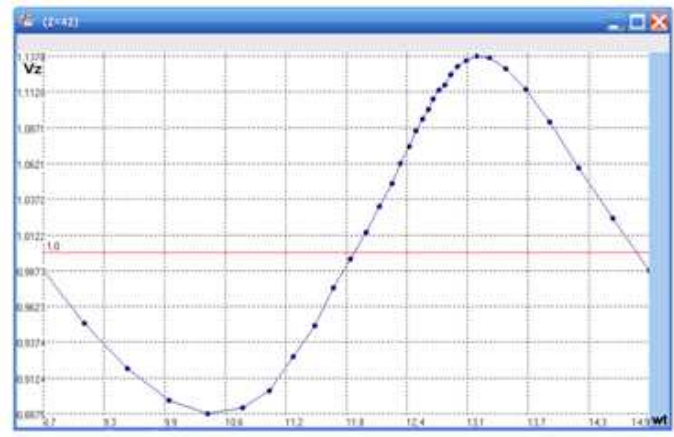

(b)

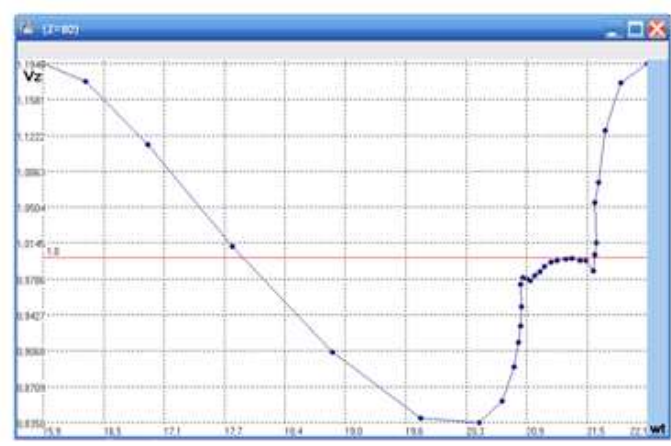

Fig 3. Relationships of longitudinal normalized velocities Vz from the phase wt (rad)

\section{Conclusion}

Investigated is change in the convection current first harmonic all over generating-amplifying klystrons of different types. At that maximum convection current first harmonic in the bunching space constitutes approximately $1.5 \mathrm{I}_{0}$ which 1.4 times exceeds the value of $1.16 \mathrm{I}_{0}$ for a normal two-resonator (double-cavity) klystron.
Shown is the possibility of operation of a double-gap resonator in the mode when the oscillations generation function is aligned with a velocity-modulation function in the phase ensuring bunching of electron bunches. In the given mode for generators with three and two double-gap resonators the value of electronic efficiency factor is gained at $50 \%$ and $55 \%$, accordingly.

The use of wide gaps in the generator section increases the efficiency factor of such a generator to $70 \%$ and does 
not require additional resonators in the buffer stage.

The dimensions and operation mode of such a device are specified.

The specified relationships allow us to make the following conclusion: in the generator with a buffer stage on one single-gap resonator electrons are properly bunched and virtually completely give off microwave energy to the output resonator gap field.

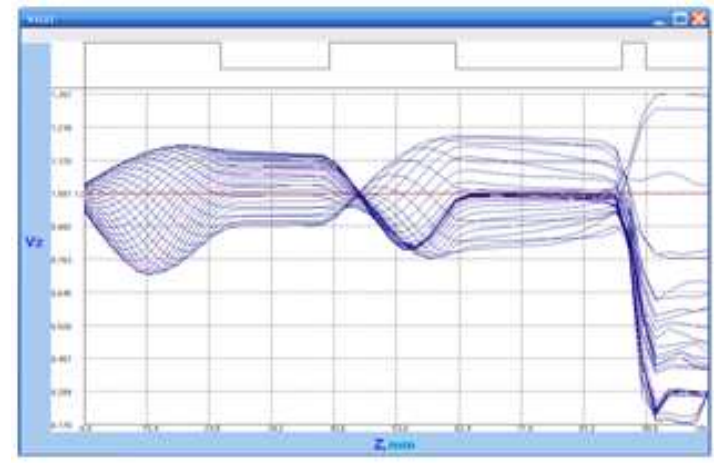

(a)

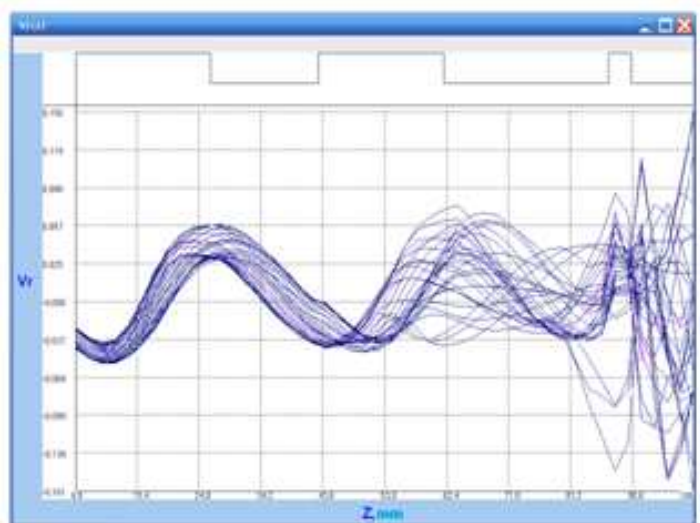

Fig 4. Relationships of longitudinal $V z$ (a) and radial $V r(b)$ normalized velocities from longitudinal coordinate $z$

\section{References}

[1] V. P. Panov et al., "About making of instruments with the big corners of flight," Herald of the RGRTU., № 2 (is. 32), 2013, pp. $110-113$.
[2] J. J. Barroso, "Split-cavity monotrons achieving 40 percent electronic efficiency" IEEE Transactions on Plasma Science. Piscataway. NJ, vol. 32, № 3, 2004, pp. 1205-1211.

[3] M. Chodorow and S. Fan, "A floating-drift-tube klystron," Proc. I. R. E., vol. 41, №1, 1953, pp. 25-31.

[4] R. B. Nelson, "A hihg-power floating-drift-tube klystron," Proc. of the 4-th Int. Congress on microwave tube. Holland. Sept. 1962, pp. 49-54.

[5] V. K. Fedyaev and O. A. Gorlin, "Efficiency factor pitron's," Radio engineering and electronics, vol. 55, № 12, 2010, pp. $1050-1055$.

[6] A. Harrison, "Klystron tubes," New York, 1947.

[7] R. Warnecke and P. Guenard, "Les Tubes electroniques a commande par modulation de vitesse," Paris, 1951.

[8] V. N. Shevchik, "Theory microwaves,” Moscow, 1959.

[9] T. S. Akimova and V. K. Fedyaev, "The double-gap cavity operation in a generating-amplifying klystrons." 201121 th Int. Crimean Conf. "Microwave \& Telecommunication Technology" (CriMiCo'2011). Sevastopol, vol 1, 2011, pp.308-309.

[10] T. S. Akimova and V. K. Fedyaev, "The double-gap resonator oscillator with a buffer stage." 2010 20th Int. Crimean Conf. "Microwave \& Telecommunication Technology” (CriMiCo'2010). Sevastopol, vol 1, 2010, pp. 269-270.

[11] V. I. Yurkin and V. K. Fedyaev, "The program of analysis of two-dimensional dynamic processes in klystrons," Vacuum and plasma electronics. RRTI. Ryazan, 1986, pp. 101-105.

[12] Yu. R. Besov et al., "Amplifying klystrons Consolidation "Svetlana"," Radio engineering, № 2, 2000, pp. 4-8.

[13] V. K. Fedyaev and I. V. Vinokurova, "The electric fields' investigation of electron bunches in trystron," 2008 Int. Conf. "Actual problems of electron devices engineering" (APEDE'2008).Saratov, 2008, pp. 58-63.

[14] T. S. Akimova, V. K. Fedyaev and O. A. Gorlin, "Condition self-excitation pitron's," Herald of the RGRTU. Electronic, № 3 (is. 33), 2010, pp. 64-68.

[15] T. S. Remizova, V. K. Fedyaev and V. I. Yurkin, "Development of the methodology calculation of devices of klystron type in self-consistent regime," 2012 Int. Conf. "Actual problems of electron devices engineering" (APEDE'2012).Saratov, 2012, pp. 91-93. 